\title{
Quantification of Tamsulosin in Human Plasma Using LC-MS/MS
}

Tummala Samba Siva Rao ${ }^{1 *}$, Rajesh Tirumala ${ }^{2 *}$ and P. Srinivas Rao ${ }^{3}$

${ }^{1}$ Department of Pharmaceutical Analysis, Vel's College of Pharmacy, Pallavaram, Chennai, TN, India

${ }^{2}$ Department of Pharmaceutical Analysis, SRM College of Pharmacy, Kattankulathur, TN-603203, India

${ }^{3}$ Department of Pharmaceutical Chemistry, Yalamarty Pharmacy College, Tarluwada, Vishakapatnam-530052, Andhra Pradesh

\begin{abstract}
A rapid and sensitive liquid chromatography tandem mass spectrometry (LC-MS/MS) method has been developed for quantitative determination of Tamsulosin (TMS). The analyte was extracted from human plasma by Liquid-Liquid Extraction (LLE) using Methyl Tertiary Butyl Ether (MTBE) and Dichloromethane (DCM). Tolteridone Tartrate (TLT) was used as the internal standard. A Phenomenex RP-18 $(50 \times 4.6 \mathrm{~mm}$ i.d., $5 \mu)$ column provided chromatographic separation of the analyte using a mobile phase containing Acetonitrile: $0.01 \mathrm{M}$ ammonium formate (pH9.0) (90:10) at a flow rate of $0.8 \mathrm{ml} / \mathrm{min}$ with an elution time as low as $3.0 \mathrm{~min}$ which was followed by detection with mass spectrometry. The Multiple Reaction Monitoring (MRM) pair (m/z) 409.3/271.4 for TMS and 326.4/147.1 for TLT. Simple isocratic chromatographic conditions and mass spectrometric detection of the method enables the detection of TMS at less than nanogram levels. The proposed method was found to be linear from 0.20-100.08 ng/ $\mathrm{ml}$. The precision and accuracy values are within 10\%. The overall recovery of TMS was $90.92 \%$.
\end{abstract}

Keywords: Tamsulosin; Tolteridone tartrate; Liquid-liquid extraction; Liquid chromatography-tandem mass spectrometry; Human plasma

\section{Introduction}

Tamsulosin hydrochloride, (2)-(R)-5-[2-[[2-(O-ethoxyphenoxy) ethyl]amino]propyl] -2-methoxy benzensulfonamide (Figure 1) hydrochloride, is a structurally new type of highly selective aadrenoceptor antagonist $[1,2]$. It has been clinically used for the urinary obstructed patients with benign prostatic hyperplasia. It is found to be more potent drug because of its low dose of 0.1-0.2 $\mathrm{mg}$ than prozosin [3], which gives a maximum plasma concentration of $7 \mathrm{ng} / \mathrm{ml}$ [4] in human. Hence it is important to develop newer analytical techniques to quantify TMS in ng levels with easy and non-tedious extraction from plasma in spite of few reported methods where the detection limit was only $0.5 \mathrm{ng} / \mathrm{ml}$ [5] which is not adequate for pharmacokinetic studies and an LC-ESI-MS method has been reported with a linearity range of $0.5-50 \mathrm{ng} / \mathrm{ml}$. Here we report an LC-MS/MS method for the quantification of TMS from human plasma with a linearity of 0.2$100.08 \mathrm{ng} / \mathrm{ml}$ with an easy extraction of the analyte which eluted in less than 2 min.

\section{Materials and reagents}

Tamsulosin (99.3\%, pure) sample was obtained from Natco Pharma, Kothur, Tolteridone tartrate was obtained from Sun Pharmaceuticals Ltd, Acetonitrile and methanol HPLC grade purchased from Qualigens, Mumbai, India. All other reagents used were Analytical Reagent grade. MilliQ water was used through out the analysis

\section{Chromatographic conditions}

The Shimadzu LC system (Shimadzu Corporation, Kyoto, Japan) is equipped with an LC-AD VP binary pump, a DGU20A5 degasser and an SIL-HTC auto sampler equipped with a CTO-10AS VP thermostated column oven. The chromatography was performed using Phenomenex RP-18, $50 \times 4.6 \mathrm{~mm}, 5 \mu \mathrm{m}$ at $40^{\circ} \mathrm{C}$ temperature. The isocratic mobile phase composition was a mixture of acetonitrile and $0.01 \mathrm{M}$ ammonium formate $(\mathrm{pH} 9.0)(90: 10 \mathrm{v} / \mathrm{v})$ at a flow rate of $0.8 \mathrm{ml} / \mathrm{min}$. Mass spectrometric detection was performed on an API 3000 triple quadrupole instrument (MDS-SCIEX, Concord, Ontario, Canada) using MRM. A turbo-ion-spray interface operating in positive ionization mode was used. Typical source conditions were as follows: the turbo-gas temperature was set at $250{ }^{\circ} \mathrm{C}$, and the ion spray needle voltage was adjusted at $5500 \mathrm{~V}$. The mass spectrometer was operated at unit resolution for both Q1 and Q3 in the MRM mode, with a dwell time of $200 \mathrm{~ms}$ per MRM channel. The precursor/product ion pairs monitored were $\mathrm{m} / \mathrm{z} 409.3-271.4$ for TMS and $\mathrm{m} / \mathrm{z} 326.4-147.1$ for the TLT. Ion source gases 1 and 2 were set at 25 and 30 (arbitrary units) respectively; the curtain gas was at 15 (arbitrary units) and the collision gas at 10 (arbitrary units). The collision energy was set at 24 for TMS and 37 for TLT, respectively. Data acquisition was performed with analyst 1.4.1 software (MDS-SCIEX, Concord, Ontario, Canada).

\section{Sample preparation}

Standard stock solutions of TMS $(1 \mathrm{mg} / \mathrm{ml})$ and the IS $(1 \mathrm{mg} /$ $\mathrm{ml}$ ) were separately prepared in a mixture (80:20) of acetonitrile and water. Working solutions for calibration and controls were prepared by appropriate dilution in acetonitrile. The IS working solution $(0.0001 \mu \mathrm{g} /$ $\mathrm{ml}$ ) was prepared by diluting its stock solution with acetonitrile. Working solutions $(0.2 \mathrm{ml})$ were added to drug-free human plasma $(9.8 \mathrm{ml})$ as a bulk, to obtain TMS concentration levels of $0.25,0.5$, $5.01,10.01,20.03,40.05,60.05,80.06$ and $100.08 \mathrm{ng} / \mathrm{ml}$ as a single batch at each concentration. Quality control (QC) samples were also prepared as a bulk on an independent weighing of standard drug, at concentrations of 0.25 (Lower Limit of Quantitation, LLOQ), 0.74 (low), 35.35 (medium) and $75.06 \mathrm{ng} / \mathrm{ml}$ (high) as a single batch at each concentration. The calibration and control bulk samples were

*Corresponding authors: Tummala Samba Siva Rao, Department of Pharmaceutical Analysis, Vel's College of Pharmacy, Pallavaram, Chennai, TN India, Tel: +91-984-898-8555; E-mail: samba.tummala9@gmail.com

Rajesh Tirumala, Department of Pharmaceutical Analysis, SRM College of Pharmacy, Kattankulathur, TN-603203, India, Tel: +91-988-527-5947; Fax: +91442-745-5717; E-mail: rajeshtirumala@hotmail.com

Received January 03, 2011; Accepted February 23, 2011; Published March 03, 2011

Citation: Samba Siva Rao T, Tirumala R, Srinivas Rao P (2011) Quantification of Tamsulosin in Human Plasma Using LC-MS/MS. J Bioanal Biomed 3: 055-058. doi:10.4172/1948-593X.1000043

Copyright: () 2011 Samba Siva Rao T, et al. This is an open-access article distributed under the terms of the Creative Commons Attribution License, which permits unrestricted use, distribution, and reproduction in any medium, provided the original author and source are credited. 
divided into aliquots in micro centrifuge tubes (Tarson, $4 \mathrm{ml}$ ) and stored in the freezer at below $-50^{\circ} \mathrm{C}$ until analysis. A plasma sample $(0.3 \mathrm{ml})$ was pipetted into a $15 \mathrm{ml}$ glass tube, then $50 \mu \mathrm{l}$ of IS working solution $(0.0001 \mu \mathrm{g} / \mathrm{ml})$ were added. After vortex mixing for $1 \mathrm{~min}, 4$ $\mathrm{ml}$ aliquot of the extraction solvent, MTBE: DCM (70:30, v/v), was added and the sample was vortex-mixed for $8 \mathrm{~min}$. The organic layer (3 $\mathrm{ml}$ ) was transferred to a glass tube and evaporated to dryness using an evaporator at $40^{\circ} \mathrm{C}$ under a stream of nitrogen. Then the dried extract was reconstituted in $200 \mu \mathrm{l}$ of acetonitrile and a $10 \mu \mathrm{l}$ aliquot was injected into the chromatographic system.

\section{Bioanalytical method validation}

A calibration curve was constructed from a blank sample (a plasma sample processed without the IS), a zero sample (a plasma processed with the IS) and nine non-zero samples covering the total range $0.25-100.08 \mathrm{ng} / \mathrm{ml}$, including the LLOQ. The calibration curves were generated using the analyte to IS peak area ratios by weighted $\left(1 / \mathrm{x}^{2}\right)$ least-squares linear regression on consecutive days. The acceptance criterion for a calibration curve was a correlation coefficient (r) of 0.99 or greater, and that each back-calculated standard concentration must be within 15\% deviation from the nominal value except at the LLOQ, for which the maximum acceptable deviation was set at $20 \%$. At least $67 \%$ of non-zero standards were required to meet the above criteria, including acceptable LLOQ and upper limit of quantification.

The within-batch precision and accuracy were determined by analyzing four sets of QC samples (LLOQ, low, medium and high concentrations) each comprising six replicates in a batch. The betweenbatch precision and accuracy were determined by analyzing five such different batches. The acceptance criteria for within- and between-batch precision were $\pm 20 \%$ for LLOQ and $\pm 15 \%$ for the other concentrations, and the accuracies were $100 \pm 20 \%$ or better for LLOQ and $100 \pm 15 \%$ or better for the other concentrations.

Recovery of TMS from the extraction procedure was determined by a comparison of the peak area of TMS in spiked plasma samples (six each of low, medium and high QCs) with the peak area of TMS in samples prepared by spiking extracted drug-free plasma samples with the same amounts of TMS at the step immediately prior to chromatography. Similarly, recovery of IS was determined by comparing the mean peak areas of extracted QC samples $(n=6)$ to mean peak areas of IS in samples prepared by spiking extracted drug-free plasma samples with the same amounts of IS at the step immediately prior to chromatography.

The stability of the analyte and IS in human plasma under different temperature and timing conditions, as well as their stability in the stock solutions, was evaluated. QC samples were subjected to short-term room temperature conditions, long-term storage conditions $\left(-50^{\circ} \mathrm{C}\right)$ and to freeze-thaw stability studies. All the stability studies were conducted at two concentration levels $(0.74$ and $75.06 \mathrm{ng} / \mathrm{ml}$ as low and high values) with six replicates for each.

\section{Results and Discussion}

\section{Mass spectrometry}

In order to develop a method with the desired LLOQ $(0.25 \mathrm{ng} / \mathrm{ml})$, it was necessary to use MS/MS detection, as MS/MS methods provide improved limit of detection and selectivity for trace-mixture analysis. The inherent selectivity of MS/MS detection was also expected to be beneficial in developing a selective and sensitive method. $[\mathrm{M}+\mathrm{H}]^{+}$was the predominant ion in the Q1 spectrum and was used as the precursor ion to obtain product ion spectra. The product ion mass spectra, and their proposed rationalizations in terms of fragmentation patterns TMS and TLT, are illustrated in Figure 2. The product ion mass spectrum of TMS showed predominant fragment ions at $\mathrm{m} / \mathrm{z} 271.1$ and 228.1.

\section{Method development}

Liquid-liquid extraction (LLE) was used for the sample preparation in this work. Six organic solvents, n-hexane, ethyl acetate, diethyl ether, dichloromethane, chloroform, t-butyl- methylether and their mixtures in different combinations and ratios were evaluated. Finally, combination of MTBE and DCM (70:30 v/v) was found to be optimal, which can produce a clean chromatogram for a blank plasma sample. The average absolute recoveries of TMS from spiked plasma samples was $78.1 \pm 1.1 \%$ and the recovery of the TLT was $54.1 \pm 1.0 \%$ at the concentration used in the assay $(0.0001 \mu \mathrm{g} / \mathrm{ml})$. Recoveries of the analytes and IS were good and it was consistent, precise and reproducible. The assay has proved to be robust in highthroughput bioanalysis. The chromatographic conditions, especially the composition of mobile phase, were optimized through several attempts to achieve good resolution and symmetric peak shapes for the analyte and TLT, as well as a short run time. It was found that a mixture of acetonitrile and 0.01M ammonium formate ( $\mathrm{pH} 9.0)(90: 10 \mathrm{v} / \mathrm{v})$ could

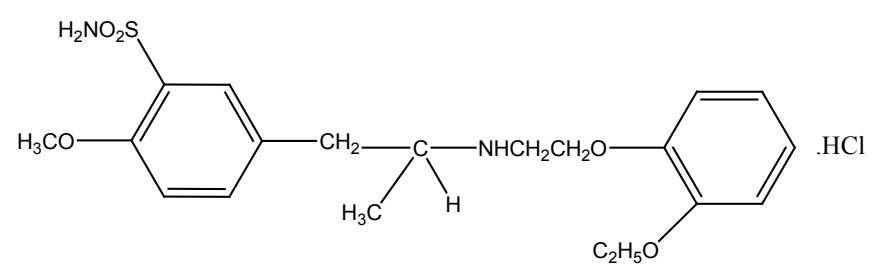

Figure 1(A): Chemical structure of Analyte (Tamsulosin).<smiles>Cc1ccc(O)c([C@@H](CCN(C(C)C)C(C)C)c2ccccc2)c1</smiles>

Figure 1(B): Chemical structure of Internal Standard (Tolteridone).

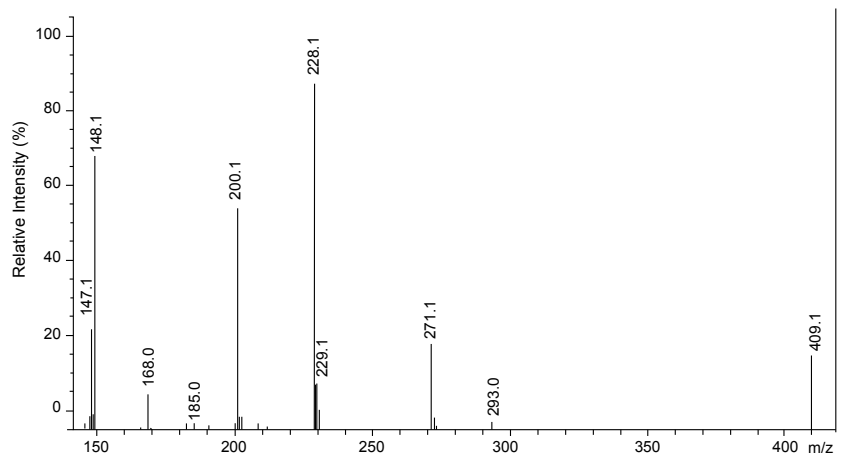

Figure 2: Mass spectra of molecular ions $\left(\mathrm{MH}^{+}\right)$of Tamsulosin. 
achieve this purpose and was finally adopted as the mobile phase. The high proportion of organic solvent eluted both the analyte and TLT at retention time of 0.93 and $1.41 \mathrm{~min}$. A flow rate of $0.8 \mathrm{ml} / \mathrm{min}$ produced good peak shapes and permitted a run time of $3.0 \mathrm{~min}$. The $\mathrm{pH}$ of the aqueous phase of the liquid chromatographic mobile phase influences both the chromatographic elution of the compounds and the formation of the $[\mathrm{M}+\mathrm{H}]^{+}$molecular ions and is strongly related to their degree of ionization. Choosing the appropriate internal standard is an important aspect to achieving acceptable method performance, especially with LC-MS/MS, where matrix effects can lead to poor analytical results. Several compounds were investigated to find a suitable IS, and finally TLT was found to be suitable. Clean chromatograms were obtained and no significant direct interferences in the MRM channels at the relevant retention times were observed. However, in ESI, signal suppression or enhancement may occur due to co-eluting endogenous components from the sample matrix. The importance of including the evaluation of matrix effect in any LC-MS/MS method is outlined in an excellent paper by Matuszewski et al. [6]. Their data strongly emphasize the need to use a blank matrix from (at least five) different sources/individuals instead of using one blank matrix pool to determine method precision and accuracy. Therefore, all validation experiments in this assay were performed with matrixes obtained from different individuals. In addition, validation experiments were performed using hemolytic and strongly lipemic matrices. As all data fall within the guidelines, we conclude that the degree of matrix effect was sufficiently low to produce acceptable data, and the method can be considered as valid.

The nine-point calibration curve was linear over the concentration range $0.25-100.08 \mathrm{ng} / \mathrm{ml}$. The calibration model was selected based on the analysis of the data by linear regression with/without intercepts and weighting factors $\left(1 / \mathrm{x}, 1 / \mathrm{x}^{2}\right.$ and none). The best linear fit and least-squares residuals for the calibration curve were achieved with a $1 / \mathrm{x}^{2}$ weighing factor, giving a mean linear regression equation for the calibration curve of $y=0.8572( \pm 0.0000) x+0.8577( \pm 0.0007)$ where ' $y$ ' is the peak area ratio of the analyte to the TLT and ' $x$ ' is the concentration of the analyte. The mean correlation coefficient of the weighted calibration curve generated during the validation was $0.9979 \pm 0.009$.

The selectivity of the method was examined by analyzing $(n=6)$ blank human plasma extract [Figure $3(\mathrm{~A})$ ] and an extract spiked only with the IS [Figure 3(B)]. As shown in Figure 3(A), no significant direct interference in the blank plasma traces was observed from endogenous substances in drug-free human plasma at the retention

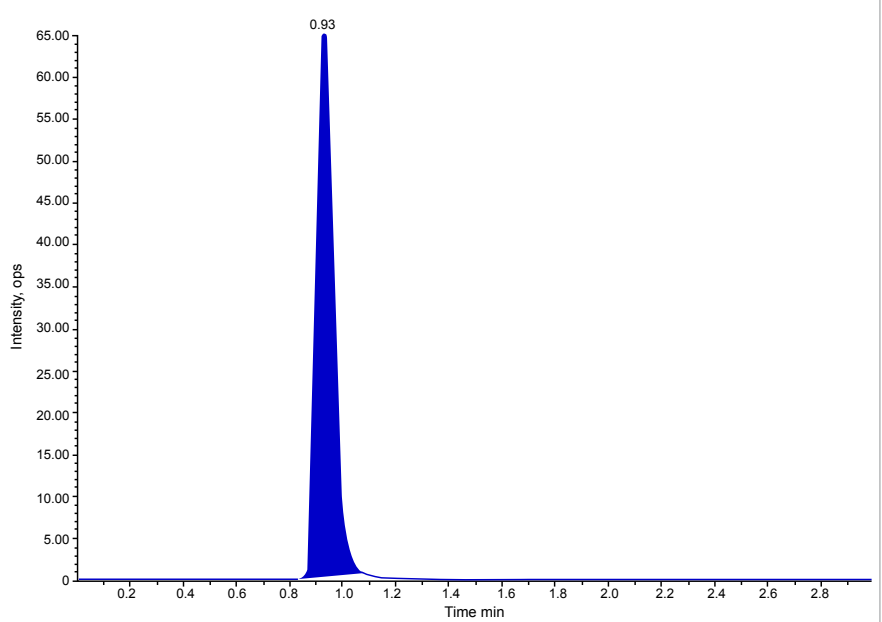

Figure 3(A): Chromatogram showing Tamsulosin $(0.93 \mathrm{~min})$ at $5 \mathrm{ng} / \mathrm{ml}$.

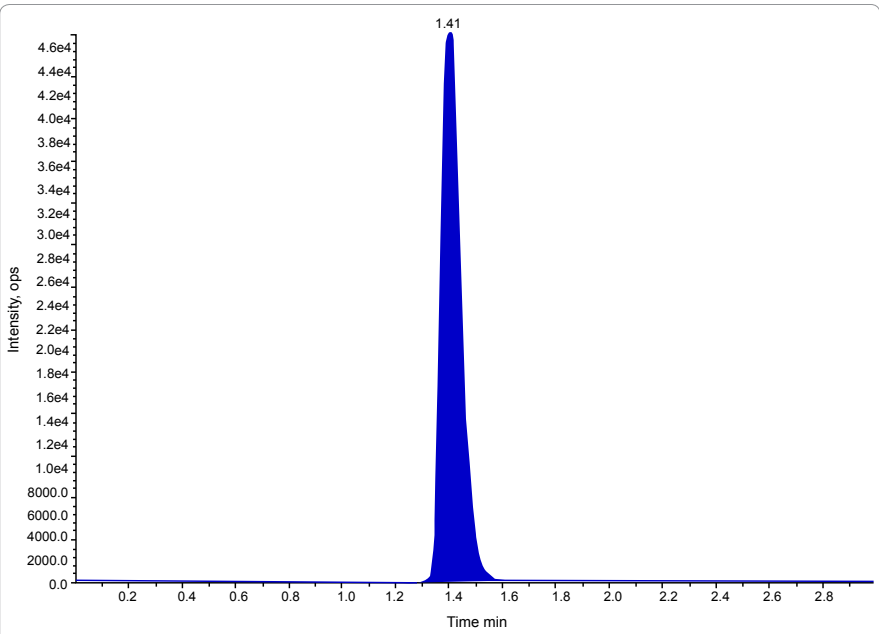

Figure 3(B): Chromatogram showing Tolteridone tartrate $(1.43 \mathrm{~min})$ at 100 $\mathrm{ng} / \mathrm{ml}$.

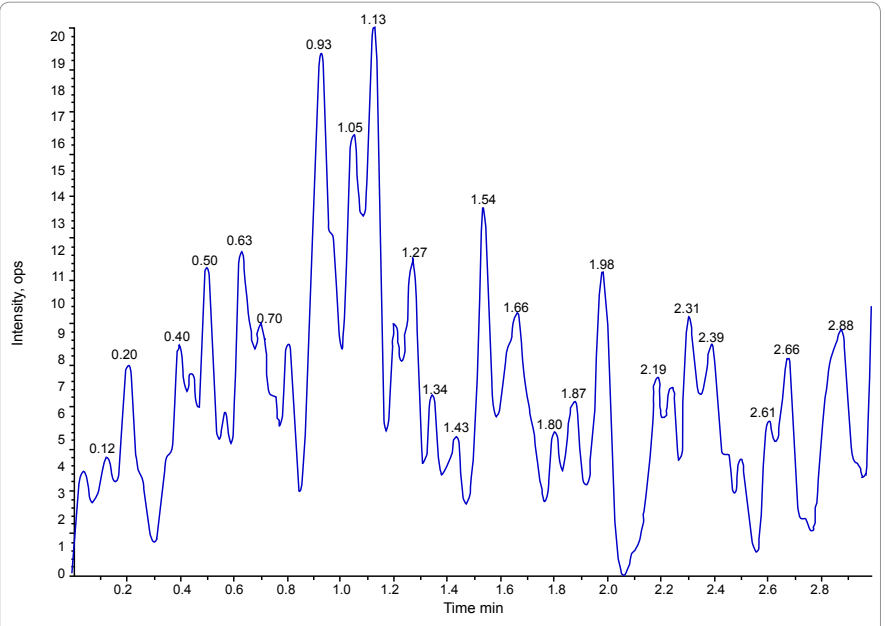

Figure 3(C): Analyte blank.

time of the analyte. Similarly, Figure 3(B) shows the absence of direct interference from the TLT to the MRM channel of the analyte. Figure $3(\mathrm{C})$ depicts a representative ion-chromatogram for the LLOQ $(0.2 \mathrm{ng} /$ $\mathrm{ml}$ ). Excellent sensitivity was observed for a $10 \mu \mathrm{l}$ injection volume; the LLOQ corresponds to ca. 3 pg on-column. The LLOQ was defined as the lowest concentration in the standard curve that can be measured with acceptable accuracy and precision and was found to be $0.2 \mathrm{ng} / \mathrm{ml}$ in human plasma. The mean response for the analyte peak at the assay sensitivity limit $(0.2 \mathrm{ng} / \mathrm{m})$ was $\sim 10$-fold greater than the mean response for the peak in eight blank human plasma samples at the retention time of the analyte. The intra-day precision and inter-day \% CV was found less than 13.45 at the selected concentrations (Table 1) of TMS.

The lower and upper quantification levels of TMS ranged from 0.74 to $75.06 \mathrm{ng} / \mathrm{ml}$ in human plasma. For the between-batch experiments the precision ranged from 3.9 to $6.7 \%$ and the accuracy from 93.6 to $98.5 \%$ (Table 1). For the within-batch experiments the precision and accuracy for the analyte met the acceptance criteria $(< \pm 15 \%)$. The upper concentration limits can be extended with acceptable precision and accuracy to $100 \mathrm{ng} / \mathrm{ml}$ by a 10 -fold dilution with control human plasma. These results suggested that samples with concentrations greater than the upper limit of the calibration curve can in this way be assayed to obtain acceptable data (Table 1). 


\begin{tabular}{|c|c|c|c|c|c|c|c|c|c|}
\hline $\begin{array}{l}\text { Quality control } \\
\text { Samples }\end{array}$ & $\begin{array}{l}\text { Nominal } \\
\text { Concentration }\end{array}$ & $\begin{array}{l}\text { Intra-assay Mean } \\
\text { concentration } \\
\text { Found }(\mathrm{ng} / \mathrm{ml})\end{array}$ & SD & $\% \mathrm{CV}$ & $\mathrm{N}$ & $\begin{array}{l}\text { Inter -assay Mean } \\
\text { Concentration } \\
\text { Found }(\mathrm{ng} / \mathrm{ml})\end{array}$ & SD & $\% \mathrm{CV}$ & $\mathrm{N}$ \\
\hline LLOQ & 0.2 & 0.212 & 0.0140 & 6.60 & 5 & 0.202 & 0.0100 & 4.95 & 5 \\
\hline LQC & 0.74 & 0.766 & 0.1041 & 13.45 & 5 & 0.732 & 0.0695 & 9.49 & 5 \\
\hline MQC & 37.3 & 37.886 & 1.7949 & 4.73 & 5 & 36.892 & 0.5955 & 1.61 & 5 \\
\hline $\mathrm{HQC}$ & 75.06 & 75.342 & 4.5505 & 6.03 & 5 & 74.002 & 2.2540 & 3.04 & 5 \\
\hline
\end{tabular}

SD: Standard Deviation; CV- Coefficient of Variance; N: Total number of observations for each oncentration; LLOQ: Lower Limit of Quantification; LQC: Lower Quality Control; MQC: Middle Quality Control; HQC: High Quality Control

Table 1: Precision studies of TMS (ng/ml).

\begin{tabular}{|c|c|c|}
\hline Sample concentration $\left(\mathrm{ng} \mathrm{mL}^{-1}\right)(n=6)$ & Concentration found (mean \pm S.D.) $\left(\mathrm{ng} \mathrm{mL}^{-1}\right)$ & $\% \mathrm{CV}$ \\
\hline \multicolumn{3}{|l|}{ Short-term stability $(1,2,3 \mathrm{~h})$} \\
\hline 0.74 & $0.726 \pm 0.1815$ & 2.51 \\
\hline 37.3 & $37.138 \pm 1.0128$ & 2.72 \\
\hline 75.06 & $75.290 \pm 2.4069$ & 3.19 \\
\hline \multicolumn{3}{|l|}{ Long-term stability (4 weeks) } \\
\hline 0.74 & $0.70 \pm 0.0220$ & 3.14 \\
\hline 37.3 & $38.004 \pm 1.9729$ & 5.19 \\
\hline 75.06 & $75.408 \pm 4.8898$ & 6.48 \\
\hline \multicolumn{3}{|l|}{ Stock Solution Stability (7, 14,21Days) } \\
\hline 0.74 & $0.762 \pm 0.0205$ & 2.69 \\
\hline 37.3 & $38.416 \pm 1.5277$ & 3.97 \\
\hline 75.06 & $75.420 \pm 1.0334$ & 1.37 \\
\hline \multicolumn{3}{|l|}{ Freeze thaw Stability (3 Cycle) } \\
\hline 0.74 & $0.718 \pm 0.0136$ & 1.89 \\
\hline 37.3 & $37.446 \pm 1.0011$ & 2.67 \\
\hline 75.06 & $75.292 \pm 2.9094$ & 3.86 \\
\hline
\end{tabular}

Table 2: Stability of plasma samples of TMS.

\section{Stability studies}

For short-term stability determination, stored plasma aliquots were thawed and kept at room temperature for a period of time exceeding that expected to be encountered during routine sample preparation (around $22 \mathrm{~h}$ ). Samples were extracted and analyzed as described above and the results indicate reliable stability behavior under the experimental conditions of the regular analytical procedure. The stability of QC samples kept in the autosampler for $22 \mathrm{~h}$ was also assessed. The results indicate that solutions of the TMS and TLT can remain in the autosampler for at least $22 \mathrm{~h}$ without showing significant loss in the quantified values, indicating that samples should be processed within this period of time.

The stability data of the analyte in plasma over three freeze-thaw cycles indicate that the analyte is stable in human plasma for three freeze-thaw cycles, when stored at below $-50^{\circ} \mathrm{C}$ and thawed to room temperature.

The long-term stability data of the analyte in human plasma stored for a period of 30 days at below $-50^{\circ} \mathrm{C}$ showed reliable stability behavior, as the mean results of the tested samples were within the acceptance criteria of $\pm 15 \%$ of the initial values of the controls. These findings indicate that storage of the analyte in plasma samples at below $-50^{\circ} \mathrm{C}$ is adequate, and no stability-related problems would be expected during routine analyses for pharmacokinetic studies.

The stability of the stock solutions was tested and established at room temperature for 6 and $22 \mathrm{~h}$, and under refrigeration $\left(4^{\circ} \mathrm{C}\right)$ for 30 days (data not shown). The results revealed optimum stability for the prepared stock solutions throughout the period intended for their daily use.

\section{Conclusion}

This assay achieved higher sensitivity and better specificity for analysis of Tamsulosin in human plasma. The LLOQ $0.2 \mathrm{ng} / \mathrm{ml}$ for Tamsulosin was better than attainable by HPLC-FL (fluorescence detection). Tolteridone tartrate proved to be a good internal standard for this assay. No significant interference caused by endogenous compounds was observed. This simple and rapid assay can be successfully used in pharmacokinetic studies of Tamsulosin in human plasma.

\section{Acknowledgements}

The author T.R is grateful to University Grants Commission for the grant of Research Fellowship.

\section{References}

1. Honda K, Nakagawa C (1986) Alpha-1 adrenoceptor antagonist effects of the optical isomers of YM-12617 in rabbit lower urinary tract and prostate. $J$ Pharmacol Exp Ther 239: 512-516.

2. Honda K, Nakagawa C, Terai, Naunyn Schmiedebergs M (1987) Further studies on (+/-)-YM-12617, a potent and selective alpha 1-adrenoceptor antagonist and its individual optical enantiomers. Naunyn Schmiedebergs Arch Pharmacol 336: 295-302.

3. Honda K, Momose N, Nakagawa C (1987) Selectivity and specificity for alpha 1-adrenoceptor blocking activity of $\mathrm{R}(-)$ - and $\mathrm{S}(+)$-YM-12617 orally administered to pithed, spontaneously hypertensive rats. J Pharm Pharmacol 39: 316-318.

4. Matsushima $\mathrm{H}$, Kamimura $\mathrm{H}$ (1998) Pharmacokinetics and plasma protein binding of tamsulosin hydrochloride in rats, dogs, and humans. Drug Metab Dispos 26: 240-245.

5. Soeishi Y, Kobori M, Kobayashi SI, Higuchi S (1990) Sensitive method for the determination of Tamsulosin in human plasma using high performance liquid chromatography with fluorescence detection. J Chromatogr B 533: 291-296.

6. Matuszewski BK, Constanzer ML, Chavez-Eng CM (2003) Strategies for the assessment of matrix effect in quantitative bioanalytical methods based on HPLC-MS/MS. Anal Chem 75: 3019-3030. 